\title{
Aspirin resistance in healthy volunteers
}

\author{
Gergely Feher*, Gabriella Pusch and Laszlo Szapary \\ Department of Neurology, School of Medicine, University of Pecs, Pecs, Hungary
}

Dear Editor,

We have read with great enthusiasm the recently published article by Marcel Tschopp and Walter $\mathrm{H}$. Reinhart entitled "Platelet aggregation under high shear conditions during and after a 28-day administration of $100 \mathrm{mg}$ acetylsalicylic acid in healthy volunteers" [1]. They concluded that platelet function as measured by the PFA-100 is not inhibited significantly after a single dose of $100 \mathrm{mg}$ aspirin, is thereafter inhibited consistently in the majority of, but not all, individuals during a 4 week treatment, and returns to normal in $48-72 \mathrm{~h}$.

This is in concordance with the findings of Perneby et al., who showed that once-daily dosing of aspirin might be insufficient in patients with increased platelet turnover [2].

On the other hand, important factors were not analyzed in their study. Previous studies demonstrated that in vitro aspirin resistance, revealed by PFA-100 CT prolongation failure, is correlated to increased plasmatic von Willebrand factor ristocetin cofactor activity, reinforcing its particular importance in PFA-100 cartridges performance [3]. The prevalence of smokers was unknown, and physical activity was also not shown, although these parameters were previously found to be associated with impaired response to aspirin $[4,5]$.

Despite of large, population-based trials, the role of haemorheological parameters is still underrepresented in circulation research and in the clinical practice. Recent publications showed the importance of impaired hemorheological parameters in the development of aspirin resistance [6].

Aspirin resistance seems to be an emerging clinical entity with potentially severe consequences [7]. By understanding the mechanism of therapeutic failure and by improving the diagnosis of this clinical entity, a new era of individualized antiplatelet therapy may arise with routine measurements of platelet activity [8]. Individualized therapy with clopidogrel seemed to be associated with better clinical outcome compared to standard treatment in a recently published multicentre study [9].

In conclusion, platelet function tests seem to be important techniques, although their widespread clinical use is substantially limited due to complex preanalytic factors, reduced specificity and reproducibility. The results of these different tests may not be comparable with each other. The definition, clinical importance and treatment of aspirin resistance should be based on the results of randomized, prospective studies.

\footnotetext{
*Corresponding author: Gergely Feher, MD, Department of Neurology, University of Pecs, Ret u. 2, H-7623 Pecs, Hungary. E-mail: gergely.feher@aok.pte.hu.
} 


\section{References}

[1] M. Tschopp and W.H. Reinhart, Platelet aggregation under high shear conditions during and after a 28-day administration of $100 \mathrm{mg}$ acetylsalicylic acid in healthy volunteers, Clin. Hemorheol. Microcirc. 38 (2008), 45-50.

[2] C. Perneby, N.H. Wallén, C. Rooney, D. Fitzgerald and P. Hjemdahl, Dose- and time-dependent antiplatelet effects of aspirin, Thromb. Haemost. 95 (2006), 652-658.

[3] T. Chakroun, G. Gerotziafas, F. Robert, C. Lecrubier, M.M. Samama, M. Hatmi and I. Elalamy, In vitro aspirin resistance detected by PFA-100 closure time: pivotal role of plasma von Willebrand factor, Br. J. Haematol. 124 (2004), 80-85.

[4] I. Coma-Canella, A. Velasco and S. Castano, Prevalence of aspirin resistance measured by PFA-100, Int. J. Cardiol. 101 (2005), 71-76.

[5] L. Christiaens, L. Macchi, D. Herpin, D. Coisne, C. Duplantier, J. Allal, G. Mauco and A. Brizard, Resistance to aspirin in vitro at rest and during exercise in patients with angiographically proven coronary artery disease, Thromb. Res. 108 (2002), 115-119.

[6] L. Mannini, R. Marcucci, R. Paniccia, E. Antonucci, C. Giglioli, S. Valente, A.M. Gori, D. Prisco, G.F. Gensini and R. Abbate, Erythrocyte deformability and white blood cell count are associated with aspirin resistance in high-risk vascular patients, Clin. Hemorheol. Microcirc. 35 (2006), 175-181.

[7] G. Krasopoulos, S.J. Brister, W.S. Beattie and M.R. Buchanan, Aspirin "resistance" and risk of cardiovascular morbidity: systematic review and meta-analysis, BMJ 336 (2008), 195-198.

[8] T.H. Wang, D.L. Bhatt and E.J. Topol, Aspirin and clopidogrel resistance: an emerging clinical entity, Eur. Heart J. 27 (2006), 647-654.

[9] L. Bonello, L. Camoin-Jau, S. Arques, C. Boyer, D. Panagides, O. Wittenberg, M.C. Simeoni, P. Barragan, F. DignatGeorge and F. Paganelli, Adjusted clopidogrel loading doses according to vasodilator-stimulated phosphoprotein phosphorylation index decrease rate of major adverse cardiovascular events in patients with clopidogrel resistance: a multicenter randomized prospective study, J. Am. Coll. Cardiol. 51 (2008), 1404-1411. 